\title{
Rib Fixation Following Trauma: A Cardiothoracic Surgeon’s Perspective
}

Ernest G Chan, Erica Stefancin and Jonathan D Cunha*

Department of Cardiothoracic Surgery, University of Pittsburgh Medical Center, Pittsburgh, PA USA

*Corresponding author: Jonathan D Cunha, Department of Cardiothoracic Surgery, University of Pittsburgh Medical Center, Pittsburgh, PA, USA, Tel: +4126486315; Fax: +4128028020; E-mail: dcunhaj@upmc.edu

Rec date: May 04, 2016; Acc date: Oct 14, 2016; Pub date: Oct 16, 2016

Copyright: $\odot 2016$ Chan EG, et al. This is an open-access article distributed under the terms of the Creative Commons Attribution License, which permits unrestricted use, distribution, and reproduction in any medium, provided the original author and source are credited.

\begin{abstract}
The most common result of blunt thoracic injury is indeed the fracture of one or more ribs. Rib fractures consists of nearly $40 \%$ of patients admitted to major trauma centers, nearly accounting for 200,000 documented cases in the national trauma databank. This injury is often used as an important indicator of trauma severity and playing a major contributing factor in as many as $50 \%$ of fatal cases. What is particularly devastating about rib fractures is their intimacy to many vital structures. The location of the rib fracture may indicate types of injuries that may occur. Patients often present with hemothorax or pneumothorax, particularly if two or more ribs are fractured.
\end{abstract}

Keywords: Rib fracture; Surgical fixation; Consensus; Hemothorax; Trauma; Injuries

\section{Introduction}

Fractures of the 4th through 9th ribs can be associated with injuries of the lung parenchyma, bronchus, and heart. Structures that may be injuried after sustaining rib fractures of the 9th rib and below includes the spleen, liver, and kidneys. Therefore, as more ribs are involved, especially with 6 or more, both pulmonary and non-pulmonary complications may ensue and need to be addressed in a timely fashion. It is not uncommon to have an associated diaphragm injury [1,2].

\section{The Pathophysiology of Rib Fractures}

The importance of the integrity of the chest wall lies with its functionality regarding the respiratory system. Much of respiratory mechanics can be described with Boyle's Law, which states that the pressure of a gas is inversely proportional to the volume of the same gas. As the diaphragm and accessory respiratory muscles contract, the chest wall achieves multidimensional expansion to increase volume. To accommodate this change in volume, a dramatic decrease in intrathoracic pressure follows as illustrated by Boyle's Law. This causes a rush of air from the atmosphere into the chest via airways and lung parenchyma to alleviate this difference in pressures.

Negative intrapleural pressure between the lung parenchyma and the parietal pleura guides the expansion and recoil of the lung parenchyma with the chest wall so they can act in a single fashion. Because of the amount of force necessary to damage integrity of the chest wall, rib fractures can be indication of trauma to the tissues and vital organs. Destabilization of the rib cage impairs spontaneous breathing mechanics substantially. This condition is exacerbated by the presence of pain. Lastly, positive intrapleural pressure may be introduced into the chest cavity through a defect in the lung or in the skin, facilitating the lung's natural tendency to recoil to a nonfunctioning state [3].

Physical injuries to the lung parenchyma may result from rib fractures as well. Sharp edges at the sight of injury can puncture organs. Bruising, tearing, and sheering of vital structures such as the lungs may occur. This direct traumatic damage to the lungs is typically coupled with a concurrent increase in vascular permeability of the lung capillaries at the sight of injury and will result in progressive respiratory failure due to the extravasation of protein rich fluids and impairment of gas diffusion at the alveolar level [4,5]. Both the combination of structural and pathophysiologic impairment of the respiratory system resulting from rib fractures can prove to be extremely morbid for patients and requires immediate intervention. The diaphragm is also at risk and a tear from a rib fracture puts the patient at risk for immediate or delayed hernia.

\section{Morbidity Following Rib Fractures}

The number of rib fractures is positively correlated to the patient's morbidity and mortality [6-8]. Flagel et al. reported a $10 \%$ mortality in patients with more than four rib fractures. This figure increased dramatically to $34 \%$ in patients presenting with eight or more fractures $[8,9]$. In addition, patients 45 years of age or older presenting with four or more rib fractures are associated with increased risk of adverse outcomes following injury [8-12]. Even after adjusting for severity of injury, comorbidity, and the presence of multiple rib fractures, patients 65 or older with simple rib fractures still were five times more likely to die compared with patients younger than 65 years [13]. Up to $30 \%$ of patients with isolated or multiple fractured ribs develop pneumonia because of their inability to breathe spontaneously as well as clear secretions $[14,15]$. This potentially lethal complication inevitably leads to longer hospital stay with the possible requirement of mechanical support which is highly associated with its own profile of complications. Therefore, underestimating the pathophysiologic potential of rib fractures, especially in the setting of high risk patients, should be avoided at all cost.

\section{Diagnosis and Evidence for Surgical Management}

Patients often present with symptoms and findings not limited to pain, point tenderness at the sight of injury, and possible crepitus. Physical examination as well as radiographic imaging are key in diagnosing rib fractures as well as identifying patients who will most benefit from surgical treatment. Initial use of chest radiography should be standard practice when evaluating patients admitted to the hospital 
Page 2 of 4

with history of trauma. The addition of CT to the evaluation process improves the sensitivity of the diagnosis of rib fracture [16].

Because of the complications associated with non-operative management, we recommend the use of surgical fixation as the treatment of older patients presenting with rib fractures that are significantly displaced as well as patients suffering from multiple ribs fractures. In 2013, the rib fracture consensus meeting was held with the purpose of creating a consensus on clinical indications for surgical rib fixation (Table 1) [17].

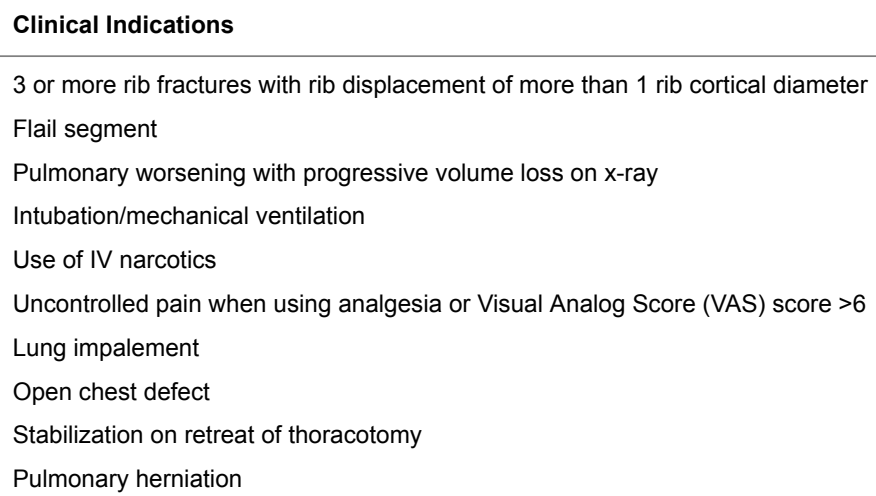

Table 1: Clinical indications for surgical rib fixation from the 2013 rib fracture consensus meeting [17].

These indications can be used as guidelines when assessing patients presenting with rib fractures as these capture most if not all of the patients with clinically significant rib fractures. However, the basis of management should always be the patient's clinical picture regardless of whether they fit these criteria or not.

Many studies have shown that the proper use of surgical treatment can steer patients away from morbid clinical outcomes. Moreover, operative rib fixation was associated with more cost-effective practices. A number of prospective trials have all shown a decrease in ICU and hospital stay, decrease in non-invasive ventilation after extubation, decrease in tracheostomy rate, decreased incidence of pneumonia, and improved spirometry at follow-up in patients who received operative management for flail chest [18-20].

Moreover, Deb et al. found that patients with a numeric pain intensity (NPI) of 7 or greater experienced a statistically significant reduction in pain postoperatively ( $8.9 \mathrm{vs} 4.7, \mathrm{p}<0.05)$ [21]. All of these findings suggest benefits of surgical treatment for the patient as well as promote cost-effective practices by decreasing resource utilization.

Even with all of these findings, concrete evidence identifying the best method for the repair of rib fractures is lacking. Certainly every rib fracture should not be treated surgically as some may heal spontaneously. Many widely accepted indications of surgical rib fixation includes flail chest, patients with consistently inadequate pain management, or have one or more symptomatic non-union rib fractures [22]. Therefore, patients that present with traumatic rib fractures require adequate assessment of both thoracic and abdominal structures in order to identify potential underlying injuries.

This can be done through physical examination, imaging modalities, and under direct visual inspection with VATS or open surgical techniques. We highly recommend considering surgical intervention in patients with multi-organ injuries sustained from many rib fractures as well as in patients in respiratory distress and are unable to be weaned from mechanical support as these are the patients who may decompensate rapidly under conservative medical management [22-24]. Figure 1 depicts such a patient with multiple rib fractures who was treated surgically with metal plates. Surgical Rib Fixation Technique

There is no shortage of surgical rib fixation techniques. The two main types of systems include the use of different types of plates and screws as well as the intra-mural nailing technique. Plates come in various materials ranging from titanium to absorbable products. These are externally fixated across areas of discontinuity and secured onto the ribs with screws. These plates may often need to be shaped to fit the contour of the injured ribs. Intra-mural nailing is an alternative to the plate and screw method.

Upon approximation of the fracture ends, these intra-mural nails are typically inserted through the cortex of the rib until they transverse the fractured location. Like with the plating system, these nails are contoured before insertion. Each has their own sets of benefits as well as profile of complications [25]. All of these techniques were created to achieve similar goals of chest wall stability leading to improvements in respiratory mechanics, proper healing of the chest wall, and decrease pulmonary restrictions associated with significant chest wall deformity.

\section{VATS Approach}

The video assisted thoracoscopic (VATS) approach to rib fixation has been proven to decrease hospital and ICU stay when compared to patients who underwent no fixation [25]. In our experience hands (JD), we feel there is a significant benefit in combining the VATS approach with rib fixation. At present, we use single or two port VATS to assess for other injuries, drain the blood which is in the chest. From a technical standpoint for rib fixation, VATS gives us added certainty of which ribs to address and localize the appropriate incision for repairing the ribs. Finally, a good rib fixation is confirmed via VATS and there is added assurance that a screw has not entered the chest such that potential lung injury could occur in the post-operative setting. Although these points are not yet widely published and proven, we firmly believe in its utility as an adjunct that adds minimal morbidity and in fact potentially affords a more favorable outcome from the procedure. 
Page 3 of 4

This is expert opinion and in the process of being analyzed in depth through our institutional experience (manuscript in preparation). Lastly, the VATS approach is associated with decreased postoperative pain compared to open approaches to various thoracic procedures [26-29]. These same VATS principles of limiting manipulation to the thoracic walls as well as minimizing access incisions will undoubtedly translate decrease pain in the postoperative setting of a patient undergoing rib fixation. Therefore, the VATS approach to rib fixation is a viable surgical approach to rib fixation and can be very beneficial when performed correctly.

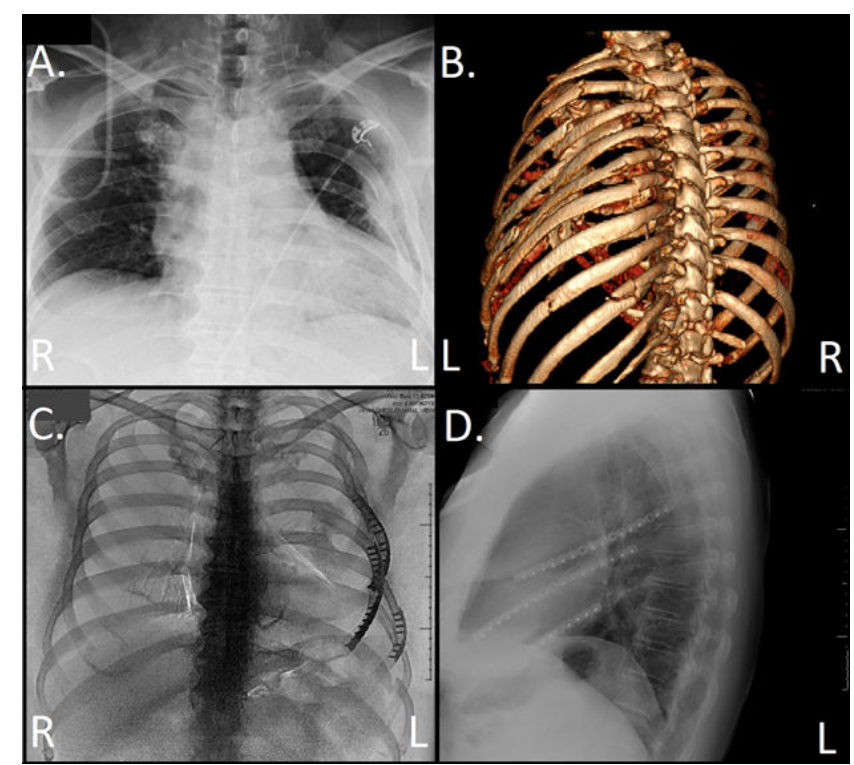

Figure 1: A: Preoperative chest $\mathrm{x}$-ray of a $67 \mathrm{y} / \mathrm{o}$ male who sustained fractures to ribs 3-10 on the left side from a fall, B: Threedimensional reconstruction of the injuries from CT imaging, $\mathrm{C}$ and $\mathrm{D}$ : Front and lateral views of postoperative chest $\mathrm{x}$-ray following surgical rib fixation with plates.

VATS surgery can be detrimental and cumbersome if not performed correctly and special considerations need to be addressed prior and during surgery. The work-up and positioning of the patient is key in preserving the benefits of this approach. As mentioned previously, chest $\mathrm{x}$-ray and CT scan will help identify the extent of injury (Figure 1A). Three-dimensional imaging may also be used but physicians should be aware that this software may smooth out fractured edges and mask smaller fractures (Figure 1B).

These tools can be used to help dictate the positioning of the patient as well as ports placement to best triangulate the areas that need repair. Reliable anatomic landmarks that may help in port placement include the mid-axillary line, the xiphoidal point of the sternum, and the jugular notch of the sternum [14]. Additional ports and small $(<8 \mathrm{~cm})$ localizing incisions performed with muscle sparing techniques may be helpful in cases with very extensive rib involvement. Postoperative imaging can be done to evaluate the surgically corrected integrity of the thoracic cavity (Figure $1 \mathrm{C}$ and $1 \mathrm{D}$ ), a practice that is routinely done in orthopedic procedures.

\section{Postoperative Management}

Because of the introduction of foreign objects into the body, careful attention is needed to possible signs of infection following surgery. Port sights and surgical incisions need to be properly addressed and monitored throughout the postoperative course. Injury sights in patients receiving metal plating need to be monitored due to the increased risk of ischemia and nerve damage associated with this technique. Pre and postoperative pain management should be common practice in the setting of a patient suffering from rib fractures to maintain both their comfort and respiratory function. Postoperatively, even with a fully functional and intact chest wall, patients may not be motivated to breathe because of the pain associated with any chest wall motion. Flexible bronchoscopy should be used liberally to prevent pneumonias as often other methods fail. Therefore, pain management is very necessary to promote spontaneous breathing even in the setting of newly approximated ribs. Vitals and oxygen saturation need to be especially monitored postoperatively to ensure adequate oxygenation in the setting of a patient with potentially compromised respiratory mechanics.

Patient education and communication is also key in this setting as their participation is necessary in the rehabilitation process. They need to be especially informed on how the postoperative course can progress and what they can do to reach the ultimate goal of being discharged with minimal morbidity. Respiratory adjuncts such as incentive spirometry should be encouraged throughout the postoperative course. Once signs of inadequate respiratory effort is identified, pain management should be reevaluated to ensure patients are optimized for recovery.

\section{Conclusion}

In conclusion, rib fractures are a common occurrence in patients experiencing trauma to the chest. Because rib fractures are highly associated with respiratory impairment as well as injuries to other thoracic structures, these patients should be monitored and properly diagnosed. Patients suitable for surgical rib repair should be quickly identified and treated. Surgical rib fixation via the VATS approach has been shown to not only decrease morbidity and mortality but also cut down on utilization of hospital resources and ultimately cost. The incidence of associated injuries that need addressing at the time of VATS is not insignificant. Rib fixation should be performed by experienced general thoracic surgeons.

\section{References}

1. Liman ST, Kuzucu A, Tastepe AI, Ulasan GN, Topcu S (2003) Chest injury due to blunt trauma. Eur J Cardiothorac Surg 23: 374-378.

2. Crawford TC, Yang KC (2015) Thoracic Trauma, in Sabiston and Spencer Surgery of the chest. In: Sellke F, Pedro J, Swanson SJ (Eds.) Elsevier Health Sciences. pp: 100-130.

3. Attinger EO, Segal MS (1959) Mechanics of breathing: The physical properties of the lung. Am Rev Respir Dis 80: 38-45.

4. Aufmkolk M, Fischer R, Voggenreiter G, Kleinschmidt C, SchmitNeuerburg KP, et al. (1999) Local effect of lung contusion on lung surfactant composition in multiple trauma patients. Crit Care Med 27: 1441-1446.

5. Cohn SM, Zieg PM (1996) Experimental pulmonary contusion: Review of the literature and description of a new porcine model. J Trauma 41: 565-571. 
Citation: Chan EG, Stefancin E, Cunha JD (2016) Rib Fixation Following Trauma: A Cardiothoracic Surgeon's Perspective. J Trauma Treat 5:

Page 4 of 4

6. Sirmali M, Türüt H, Topçu S, Gülhan E, Yazici U, et al. (2003) A comprehensive analysis of traumatic rib fractures: morbidity, mortality and management. Eur J Cardiothorac Surg 24: 133-138.

7. Sharma OP, Oswanski MF, Jolly S, Lauer SK, Dressel R, et al. (2008) Perils of rib fractures. Am Surg 74: 310-314.

8. Flagel BT, Luchette FA, Reed RL, Esposito TJ, Davis KA, et al. (2005) Half-a-dozen ribs: the breakpoint for mortality. Surgery 138: 717-725.

9. de Jong MB, Kokke MC, Hietbrink F, Leenen LP (2014) Surgical management of rib fractures: strategies and literature review. Scand J Surg 103: $120-125$.

10. Holcomb JB, McMullin NR, Kozar RA, Lygas MH, Moore FA (2003) Morbidity from rib fractures increases after age 45. J Am Coll Surg 196: 549-555.

11. Kent R, Woods W, Bostrom O (2008) Fatality risk and the presence of rib fractures. Ann Adv Automot Med 52: 73-82.

12. Todd SR, McNally MM, Holcomb JB, Kozar RA, Kao LS, et al. (2006) A multidisciplinary clinical pathway decreases rib fracture-associated infectious morbidity and mortality in high-risk trauma patients. Am J Surg 192: 806-811.

13. Bergeron E, Lavoie A, Clas D, Moore L, Ratte S, et al. (2003) Elderly trauma patients with rib fractures are at greater risk of death and pneumonia. J Trauma 54: 478-485.

14. Bemelman M, Baal M, Yuan JZ, Leenen L (2016) The role of minimally invasive plate osteosynthesis in rib fixation: A review. Korean J Thorac Cardiovasc Surg 49: 1-8.

15. Bulger EM, Arneson MA, Mock CN, Jurkovich GJ (2000) Rib fractures in the elderly. J Trauma 48: 1040-1047.

16. Livingston DH, Shogan B, John P, Lavery RF (2008) CT diagnosis of rib fractures and the prediction of acute respiratory failure. J Trauma 64 905-911.

17. Diaz J, Shiroff AM, Gasparri M, Lottenberg L, Pohlman T, et al. (2013) Special report: Integrating surgical rib fixation into clinical practice: A report from the rib fracture consensus meeting. General Surgery News.

18. Marasco SF, Davies AR, Cooper J, Varma D, Bennett V, et al. (2013) Prospective randomized controlled trial of operative rib fixation in traumatic flail chest. J Am Coll Surg 216: 924-932.
19. Granetzny A, Abd El-Aal M, Emam E, Shalaby A, Boseila A (2005) Surgical versus conservative treatment of flail chest. Evaluation of the pulmonary status. Interact Cardiovasc Thorac Surg 4: 583-587.

20. Tanaka H, Yukioka T, Yamaguti Y, Shimizu S, Goto H, et al. (2002) Surgical stabilization of internal pneumatic stabilization? A prospective randomized study of management of severe flail chest patients. J Trauma 52: 727-732

21. Deb J (2014) Rib fracture stabilization reduces chest wall pain following blunt thoracic trauma. J Trauma Treat S2: 004.

22. Nirula R, Allen B, Layman R, Falimirski ME, Somberg LB (2006) Rib fracture stabilization in patients sustaining blunt chest injury. Am Surg 72: 307-309.

23. Campbell N, Conaglen P, Martin K, Antippa P (2009) Surgical stabilization of rib fractures using Inion OTPS wraps-techniques and quality of life follow-up. J Trauma 67: 596-601.

24. Mayberry JC, Terhes JT, Ellis TJ, Wanek S, Mullins RJ (2003) Absorbable plates for rib fracture repair: Preliminary experience. JTrauma 55: 835-839.

25. Tarng YW, Liu YY, Huang FD, Lin HL, Wu TC, et al. (2016) The surgical stabilization of multiple rib fractures using titanium elastic nail in blunt chest trauma with acute respiratory failure. Surg Endosc 30: 388-395.

26. Bendixen M, Jørgensen OD, Kronborg C, Andersen C, Licht PB (2016) Postoperative pain and quality of life after lobectomy via video-assisted thoracoscopic surgery or anterolateral thoracotomy for early stage lung cancer: a randomised controlled trial. Lancet Oncol 17: 836-844.

27. Villamizar NR, Darrabie MD, Burfeind WR, Petersen RP, Onaitis MW, et al. (2009) Thoracoscopic lobectomy is associated with lower morbidity compared with thoracotomy. J Thorac Cardiovasc Surg 138: 419-425.

28. Barbour AP, Mc Cormack OM, Baker PJ, Hirst J, Krause L, et al. (2016) Long-term health-related quality of life following esophagectomy: A nonrandomized comparison of thoracoscopically assisted and open surgery. Ann Surg.

29. Detterbeck F (2009) Thoracoscopic versus open lobectomy debate: the pro argument. Thorac Surg Sci 6: 4 